\title{
What Are the Variables Associated With Altmetric Scores? - An Overview of Methodological Reviews
}

Amanda Costa Araujo ( $\square$ mandaa_costa@hotmail.com )

Masters and Doctoral Programs in Physical Therapy, Universidade Cidade de São Paulo. https://orcid.org/0000-0003-2740-8252

Adriane Aver Vanin

Universidade Cidade de Sao Paulo

Dafne Port Nascimento

Universidade Cidade de Sao Paulo

Gabrielle Zoldan Gonzalez

Universidade Cidade de Sao Paulo

Leonardo Oliveira Pena Costa

Universidade Cidade de Sao Paulo

\section{Research}

Keywords: Altmetric, Altmetrics, social impact, social media, methodological review

Posted Date: December 18th, 2020

DOl: https://doi.org/10.21203/rs.3.rs-128288/v1

License: (c) (i) This work is licensed under a Creative Commons Attribution 4.0 International License.

Read Full License 


\section{Abstract}

Background: Currently, social media has been used to disseminate contents of scientific articles. In order to measure this type of impact a new tool named Altmetric was created. Altmetric aims to quantify the impact of each article through the media online. This overview of methodological reviews aims to describe the associations between the publishing journal and the publishing articles variables with Altmetric scores.

Methods: Search strategies on MEDLINE, EMBASE, CINAHL, CENTRAL and Cochrane Library. We extracted data related to the publishing trial and the publishing journal associated with Altmetric scores.

Results: A total of 11 studies were considered eligible. These studies summarized a total of 565,352 articles. The variables citation counts, journal impact factor, access counts, papers published as open access and press release generated by the publishing journal were associated with Altmetric scores. The magnitudes of these correlations ranged from weak to moderate.

Conclusion: Citation counts and journal impact factor are the most common associators of high Altmetric scores. Other variables such as access counts, papers published in open access journals and the use of press releases are also likely to influence online media attention.

\section{Systematic Review registrations: Not applicable}

\section{Background}

The most common way to assess the impact of an article is based upon the number of citations[1]. Moreover, the mean number of citations for all the articles published in the journal in the preceding two years is named journal's impact factor[1]. However, the number of citations and the journal's impact factor do not precisely reflect if the message of the paper is reaching a wider audience[2]. Currently, social media has been used to disseminate contents of scientific articles[3,4]. However until recently, the impact of scientific articles on social media was not quantified. In order to measure this type of impact a new score (named as Altmetric) was created[3, 4].

Altmetric measures the impact of each article through the attention attracted online[3]. Moreover, the Altmetric score reveals the instantaneous attention attracted online of the articles in news outlets, commentaries on blogs, number of tweets and mentions on social media. There are two types of Altmetric scores. The Altmetric mentioned score includes data sources involving social media (e.g. Facebook, Twitter); newspapers, encyclopaedias (e.g. Wikipedia); online platforms (e.g. Faculty1000 and Publication Peer-Reviews); videos on YouTube; sites on questions and answers (e.g. Q\&A stack overflow) and policy documents on PDF documents available over the internet. The Altmetric reader score includes data sources involving reference managers available online (e.g. Mendeley, CiteULike and Connotea).

Research about Altmetric is increasing and becoming more popular in the recent years[5]. However, most articles about Altmetric published to date are only introductory tutorials or editorials[1, 3, 4, 6, 7]. Patthi et 
al[2] published a systematic review in the field of dentistry that aimed to analyze the correlations between journal citations and Altmetric scores. This review concluded that journal citations and Altmetric scores are positively correlatated (with pearson's $r$ ranging from 0.30 to 0.61 ).

Recent articles from several research fields[8-12], showed that the number of citations of articles and Altmetric are positively associated. Finch et al.[10] showed that the number of tweets (i.e. an Altmetric component) could predict citations within the first 3 days of article publication. Araujo et al.[13] found that number of citations and journal's impact factor were positively associated with Altmetric[13]. These authors also found that the number of years since publication and having a descriptive title (title describes the aim, but does not reveal the main conclusions) were negatively associated with Altmetric[13]. Therefore, it is assumed that the publishing journal and the publishing articles variables, for example citation counts, journal impact factor, access counts (i.e. considered as the sum of HTML views and PDF downloads), papers published as open access, time since publication and press release generated by the publishing journal are likely to be associated with the Altmetric[13]. This overview of methodological reviews aims to summarize all available evidence on the associations between the publishing journal and the publishing articles variables with Altmetric scores.

\section{Methods}

\subsection{Research Question}

What are the publishing journal and the publishing articles variables associated with Altmetric scores?

\subsection{Search strategy for identification of studies}

Systematic searches were conducted on MEDLINE, EMBASE, CINAHL, CENTRAL and Cochrane Library, including publications since the inception of these databases until July 3, 2018 without language restrictions. We used two search terms (Altmetric OR Altmetrics) in all databases.

\subsection{Inclusion criteria}

We included any original research article that measured any type of association between the publishing journal and the publishing article with Altmetric scores, such as: citation counts (i.e. number of citations), journal impact factor, access counts (i.e. considered as the sum of HTML views and PDF downloads), papers published as open access, time since publication and press release generated by the publishing journal.

\subsection{Exclusion Criteria}

Letters to the editor, editorials and conference abstracts were excluded.

\subsection{Data collection}

Two review authors (AA and AV) independently screened all studies for eligibility and data extraction. All discrepancies identified during the stages and throughout the review were resolved via discussion, or by 
arbitration provided by another investigator (DN). The study selection process included: (1) screening the titles and abstracts and (2) screening of full text articles.

\subsection{Data extraction}

Two review authors independently extracted the following data: (1) authors; (2) year of publication; (3) research field; (4) sample size of articles analised; (5) study designs of the included articles; (6) aims of the article; (7) study results; (8) study conclusions. Variables about the publishing journal of articles included: (9) journal impact factor; (10) access counts (i.e. considered as the sum of HTML views and PDF downloads); (11) papers published as open access and (12) press release generated by the publishing journal. Variables about the publishing articles included: (13) citation counts (i.e. number of citations) and (14) time since publication. We also collected data related to (15) the Altmetric mentioned score and (16) the Altmetric reader score. We contacted authors by email to request the any information that was not reported in the original manuscripts.

\subsection{Ethics and registration}

No ethical approval was required for this type of study. As this review has no clinical outcomes, no registration was needed.

\subsection{Data analysis}

In this study was not possible to perform a meta-analysis of the studies due to the large heterogeneity. For this reason, our results were reported descriptively.

\section{Results}

\subsection{Search results}

The initial electronic database search yielded a total of 334 potentially eligible studies. After screening by title and abstract and removal of duplicates, we considered 22 potentially eligible studies for inclusion and retrieved full-text articles. Eleven published studies[12-22] met the inclusion criteria and were included in this review. The study flow diagram of the eligibility assessment is presented in FIGURE 1.

\subsection{Characteristics of included studies}

A total of 11 studies were considered eligible. These studies summarized a total of 565,352 articles. Eligible studies were published between 2014 and 2018. The study design of the included articles were mixed research designs[12,14-22] and randomized controlled trials in low back pain[13]. The research fields from these articles include Medical Education[14], Physiotherapy[13], Emergency Medicine[21], Biomedical Area[15], Multidisciplinary[16], Dentistry[22], Social Sciences \& Humanities, Engineering \& Technology, Medical \& Natural Sciences[17], Oncology[18], Solid Organ Transplantation[19], Ecology and Conservation[20] and Radiology[12]. The main objective of the included studies was assessing the association with Altmetric scores and the variables: citation counts (i.e. number of citations), journal 
impact factor, access counts (i.e. considered as the sum of HTML views and PDF downloads), papers published as open access, time since publication and/or press release generated by the publishing journal. The summary of the methods, data analysis, results and conclusions are presented in the TABLE 1.

\subsection{Statistical analysis and associations of included studies}

Different types of analyses were performed in the included studies: correlation analysis[12,14,16,19,21,22], multivariable regression analysis[13,18], boosted regression trees analysis[20], principal component analysis and factor analysis[15,17]. The main results of the included studies demonstrated that the variables citation counts (i.e. number of citations), journal impact factor, access counts (i.e. considered as the sum of HTML views and PDF downloads), papers published as open access, time since publication and press release generated by the publishing journal were associated with Altmetric scores. The magnitude of these associations ranged from weak to moderate (TABLE 2).

\section{Discussion}

The objective of this overview of methodological reviews was to summarize all available evidence on the associations between the publishing journal and the publishing articles variables with Altmetric scores. We found that citation counts (i.e. number of citations), journal impact factor, access counts (i.e. considered as the sum of HTML views and PDF downloads), papers published as open access, time since publication and press release generated by the publishing journal were associated with Altmetric scores. The magnitude of these associations ranged from weak to moderate. Besides that, we observed that citation counts or journal impact factor were associated with Altmetric scores in all included studies[1222].

There is a previous systematic review about the correlation between citations counts and Altmetric in medical research[2]. Besides that, there are articles that have measured associations between the citation counts and Altmetric scores[12, 14]. According with the systematic review[2] and these articles[12, 14] there is a positive correlation between citation counts and Altmetric scores. Similarly, our overview found positive associations (ranged from weak to moderate) between citation counts and Altmetric scores[1222]. These results are similar to the ones related to journal's impact factor[13]. This is no unexpected, since the journal's impact factor is based on citations counts of scientific articles[1].

We also found that most included articles, with the exception of Araujo et al[13] and Knight[19], did not analyze the Altmetric reader score. In this way, our findings are largely are based on Altmetric mentioned score. We strongly recommend that further investigations on Altmetric reader score should be conducted.

There are no studies that have identified specific characteristics of articles, for example, analysis on studies that published popular/hot topics (e.g. studies on zika virus, miraculous diets, cancer prevention, early life in earth, religious evidence). Moreover, there are no analysis of studies comparing if the direction 
of the results (i.e. positive versus negative conclusions) influences Altmetric scores. These characteristics are likely to increase the number of access and sharing these articles on social media[13]. We recommend that future studies should identify if these characteristics are associated with Altmetric scores.

The major strength of this study is about the inclusion of studies from all fields of the research $(n=$ 565,352 articles analised). On the other hand, a possible limitation of this study is about the large heterogeneity of the included studies. Because of this, the data was analised only descriptively. The Altmetric is still a new topic and there are just few avaliable studies.

\section{Conclusion}

Citation counts, journal impact factor, access counts (i.e. considered as the sum of HTML views and PDF downloads), papers published as open access, time since publication and press release generated by the publishing journal were associated with Altmetric scores. However, more research is needed to confirm the results obtained in this overview of methodological reviews.

\section{List Of Abbreviations}

Not applicable.

\section{Declarations}

\section{Ethical Approval and Consent to participate}

Not applicable.

\section{Consent for publication}

Not applicable.

\section{Availability of data and materials}

The datasets used and/or analysed during the current study are available from the corresponding author on reasonable request.

\section{Competing interests}

The authors declare that they have no competing interests.

\section{Funding}

Not applicable.

\section{Authors' contributions}


A.A, A.V collected the data and performed the analysis. L.C supervised the project. A.A, A.V, D.N, G.Z and L.C wrote the manuscript.

\section{Acklowdgements}

Not applicable.

\section{References}

1. Dinsmore A, Allen L, Dolby K. Alternative Perspectives on Impact: The Potential of ALMs and Altmetrics to Inform Funders about Research Impact. PLOS Biol 2014;12(11):1-4. PMID:25423184

2. Patthi B, Prasad M, Gupta R, Singla A. Altmetrics - A Collated Adjunct Beyond Citations for Scholarly Impact: A Systematic Review. J Clin Diagnostic Res 2017;11(6):16-20. PMID:28764311

3. Brigham T. An Introduction to Altmetrics. Med Ref Serv Q 2014;33(4):438-447. PMID:25316077

4. Melero R. Altmetrics- a complement conventional metrics. Biochem Medica 2015;2(25):152-160. PMID:26110028

5. Araujo AC, Nascimento DP, Gonzalez GZ, Oliveira L, Costa P. How to increase the visibility of scientific articles through social media? Braz J Phys Ther [Internet] Associação Brasileira de Pesquisa e PósGraduação em Fisioterapia; 2018;22(6):435-436. [doi: 10.1016/j.bjpt.2018.08.009]

6. Rhee JS. High-Impact Articles - Citations , Downloads, and Altmetric Score. JAMA Facial Plast Surg 2015;17(5):1-2. PMID:26226501

7. Fabry G. Beyond the Impact Factor - What do alternative metrics have to offer? GMS J Med Educ 2017;34(2):1-8. PMID:28584875

8. Eysenbach G. Can Tweets Predict Citations? Metrics of Social Impact Based on Twitter and Correlation with Traditional Metrics of Scientific Impact. J Med Internet Res 2011;14(1):e123. PMID:22173204

9. Pinho-Costa L, Yakubu K, Hoedebecke K, Laranjo L, Reichel CP, Colon-Gonzalez M del C, Neves AL, Errami $\mathrm{H}$. Healthcare hashtag index development: Identifying global impact in social media. $\mathrm{J}$ Biomed Inform 2016;63(1):390-399. PMID:27645323

10. Finch T, O'Hanlon N, Dudley SP. Tweeting birds: online mentions predict future citations in ornithology. R Soc Open Sci 2017;4(11):1-11. [doi: 10.1098/rsos.171371]

11. Liu C, Xu YQ, Wu H, Chen SS, Guo JJ. Correlation and Interaction Visualization of Altmetric Indicators Extracted From Scholarly Social Network Activities: Dimensions and Structure. J Med Internet Res 2013;15(11):1-17. PMID:24275693

12. Rosenkrantz AB, Ayoola A, Singh K, Jr RD. Alternative Metrics ("Altmetrics") for Assessing Article Impact in Popular General Radiology Journals. Acad Radiol 2017;24(7):1-7. PMID:28256440

13. Araujo AC, Nascimento DP, Gonzalez GZ, Maher CG, Costa LOP. Impact of Low Back Pain Clinical Trials Measured by the Altmetric Score: Cross-Sectional Study. J Med Internet Res 2018;20(4):1-9. [doi: 10.2196/jmir.9368] 
14. Amath A, Ambacher K, Leddy JJ, Wood TJ, Ramnanan CJ. Comparing alternative and traditional dissemination metrics in medical education. Med Educ 2017;51(1):1-7. PMID:28719136

15. Bornmann L, Haunschild R. Do altmetrics correlate with the quality of papers? A large-scale empirical study based on F1000Prime data. PLoS One 2018;13(5):1-12. [doi: doi.org/10.1371/journal.pone.0197133]

16. Costas R, Zahedi Z, Wouters P. Do "Altmetrics" Correlate With Citations? Extensive Comparison of Altmetric Indicators With Citations From a Multidisciplinary Perspective. J Assoc Inf Sci Technol 2015;66(10):2003-2019. [doi: 10.1002/asi]

17. Didegah F, Bowman TD, Holmberg K. On the Differences Between Citations and Altmetrics: An Investigation of Factors Driving Altmetrics Versus Citations for Finnish Articles. J Assoc Sci Tecnol 2018;69(6):832-843. [doi: 10.1002/asi.23934]

18. Haneef R, Ravaud P, Baron G, Ghosn L, Boutron I. Factors associated with online media attention to research: a cohort study of articles evaluating cancer treatments. Res Integr Peer Rev Research Integrity and Peer Review; 2017;2(9):1-8. [doi: 10.1186/s41073-017-0033-z]

19. Knight SR. Social Media and Online Attention as an Early Measure of the Impact of Research in Solid Organ Transplantation. Transplantation 2014;98(5):490-496. [doi: 10.1097/TP.0000000000000307]

20. Lamb CT, Gilbert SL, Ford AT. Tweet success? Scientific communication correlates with increased citations in Ecology and Conservation. PeerJ Life Evironment 2018;12(6):1-14. [doi: 10.7717/peerj.4564]

21. Barbic D, Tubman M, Lam H, Barbic S. An Analysis of Altmetrics in Emergency. Acad Emerg Med 2016;23(3):251-268. [doi: 10.1111/acem.12898]

22. Delli K, Livas C, Spijkervet F, Vissink A. Measuring the social impact of dental research: An insight into the most influential articles on the Web. Oral Dis 2017;23(8):1155-1161. PMID:27935037

\section{Tables}

TABLE 1. Summary of the studies 


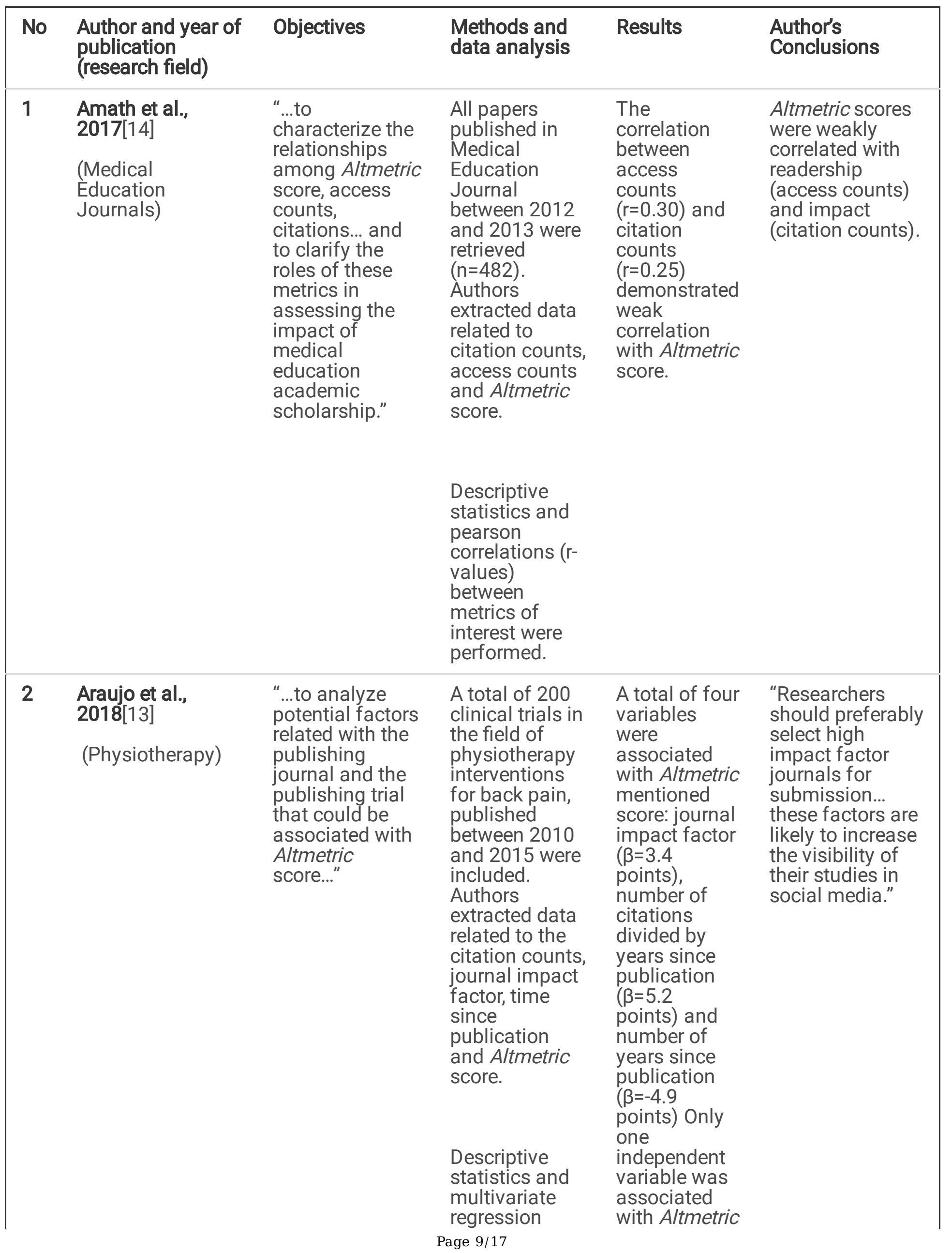


models were performed.

reader score:

number of

citations

divided by

years since

publication

( $\beta=10.1$

points).

\section{Barbic et al., 2016[21]}

(Emergency Medicine)
"... to describe the traditional metrics and Altmetric scores of the 50 most frequently cited articles

published in emergency medicine (EM) journals. Since many articles related to EM are published in other journals, the secondary aim of this study was to describe the Altmetric scores of the most frequently cited articles relevant to EM in other biomedical

journals."
The most

frequently

cited articles in

the top 10

Emergency

Medicine

journals were

included. In

total 50 papers

were included.

Authors

extracted on

citation counts,

journal impact

factor and

Altmetric

score.

Descriptive

statistics and

spearman

correlations ( $r$ -

values) were

performed.
Authors

observed a

correlation

between

citation

counts

$(r=0.22)$ and

journal

impact factor

$(r=0.35)$ with

Altmetric

score.
The study demonstrated that there is weak correlation between citation counts and journal impact factor with Altmetric scores for the top papers in Emergency Medicine and other biomedical journals.

\section{Bornmann et al., 2018[15]}

(Biomedical Area)

“...to analyze the
dimensions of
measurement
for traditional
metrics (citation
counts) and
Altmetric by
using principal
component
analysis (PCA)
and factor
analysis (FA)..."

A search in F1000Prime and Altmetric dataset between 2011 and 2013 was conducted. In total 33.683 papers were included. Authors extracted data on citation counts and Altmetric score.

Principal component analysis (PCA), factor analysis “...the results of the PCA and $F A$ showed that Altmetric score operate along different dimensions, whereas Mendeley counts are related to citation counts..." 
(FA) and

regression

models were

performed.

$5 \quad$ Costas et al., 2015[16]

(Multidisciplinary)
To determine In total

the relationship

between citation

counts, journal

impact factor

and Altmetric

score.
500.229

papers were

included.

Authors

extracted data

on citation

counts, journal

impact factor

and Altmetric

score.

\author{
Authors \\ observed a \\ correlation \\ between \\ citation \\ counts \\ $(r=0.18)$ and \\ journal \\ impact factor \\ $(r=0.19)$ with \\ Altmetric \\ score.
}

"...the weak

correlation with

citations

suggests that the potential of

Altmetric to

replace

traditional

citation analysis

is not very

strong..."

Descriptive

statistics and

spearman

correlations ( $r$ -

values) were

performed.

\section{Delli et al., 2017[22]}

(Dentistry)

\begin{abstract}
"To identify the most discussed dental articles on the Web and to assess the association between Altmetric score, publication characteristics and citations."
\end{abstract}

\begin{tabular}{|c|c|c|}
\hline $\begin{array}{l}\text { A search in } \\
\text { Altmetric } \\
\text { dataset for } \\
\text { articles } \\
\text { published in } \\
\text { the } 91 \text { dental } \\
\text { journals in } \\
2015 \text { was } \\
\text { performed. In } \\
\text { total } 100 \\
\text { articles with } \\
\text { the highest } \\
\text { online } \\
\text { attention } \\
\text { dental journals } \\
\text { were included. } \\
\text { Authors } \\
\text { extracted data } \\
\text { on citation } \\
\text { counts, time } \\
\text { since } \\
\text { publication } \\
\text { and Altmetric } \\
\text { score. }\end{array}$ & $\begin{array}{l}\text { The } \\
\text { correlation } \\
\text { between days } \\
\text { since } \\
\text { publication of } \\
\text { articles } \\
(\mathrm{r}=-0.25) \text { with } \\
\text { Altmetric } \\
\text { score } \\
\text { demonstrated } \\
\text { negative and } \\
\text { weak } \\
\text { correlation. } \\
\text { The } \\
\text { correlation } \\
\text { between } \\
\text { citations } \\
\text { counts } \\
\text { ( } r=0.27) \\
\text { demonstrated } \\
\text { positive and } \\
\text { weak } \\
\text { correlation } \\
\text { with Altmetric } \\
\text { score. }\end{array}$ & $\begin{array}{l}\text { "Increased social } \\
\text { impact of dental } \\
\text { articles is not } \\
\text { significantly } \\
\text { associated with } \\
\text { high citation } \\
\text { rates." }\end{array}$ \\
\hline
\end{tabular}

Descriptive statistics, Kruskal-Wallis test and spearman correlations ( $r-$ 
values) were

performed.

7 Didegah et al., 2018[17]

(Social Sciences

\& Humanities,

Engineering \&

Technology,

Medical \& Natural

Sciences)
To analyze the differences between citation counts and Altmetric factors.
All papers

indexed by the

Web of

Science by

selecting

Finland as the country and publishing years between 2012 and 2014 were performed. In total 13.623 papers were included.

Authors

extracted data

on citation counts and Altmetric score. “...journal impact factor... are the two factors that significantly associate with increased citation counts and with all Altmetric scores."
Altmetric reader score are associated to citation counts.

Factor analysis

was

performed.

\section{Haneef et al., 2017[18]}

(Oncology)

"...to describe
and identify the
factors
associated with
online media
attention of
articles
evaluating
cancer
treatments."

The search in

PubMed

published

during the first

6 months of

2014 in

oncology and

medical

journals was

performed. In

total 792

papers were

included.

Authors

extracted data

on journal

impact factor,

press release,

open access

and Altmetric

score.

\section{Factors} associated with high

Altmetric score were presence of a press release (RoM=10.14, 95\% Cl [4.9120.96]), open access articles (RoM=1.48, 95\% Cl [1.02$2.16])$, and journal impact factor (RoM=1.10, 95\% Cl [1.071.12].
"Most important factors associated with high online media attention were the presence of a press release and the journal impact factor..."
Descriptive statistics, regression analysis and regression 
coefficients

represent the

logarithm of

ratio of mean

(RoM) were

performed.

\section{Knight, 2014[19]}

(Solid Organ Transplantation)

"...to measure
the current use
of alternative
metrics... and to
examine the
association
between
Altmetric score
and traditional
citation
measures in the
field of solid
organ
transplantation."

The search in

MEDLINE with

subject

headings

relating to

solid organ

transplantation

between

August 2011

and July 2012

was

performed. In

total 6.979

articles were

included.

Authors

extracted data

on citation

counts and

Altmetric

score.
The

correlation

between

citation

counts

$(r=0.16)$ with

Altmetric

mentioned

score

demonstrated

weak

correlation.

The

correlation

between

citation

counts

$(r=0.23)$ with

Altmetric

reader score

demonstrated

weak

correlation.
"Social media

and online

attention act as

early predictors

of the impact of

transplant

research as

measured by

later citation rate.

Blogging and

expert

recommendation, in particular, are associated with higher citation rates."
Descriptive

statistics,

Wilcoxon

Rank-sum test and spearman correlations ( $r$ values) were performed.

\section{Lamb et al.,} 2018[20]

(Ecology and Conservation)

"...to measure
the association
between citation
rates and the
Altmetric score -
an indicator of
the amount and
reach of the
attention an
article has
received - along
with other forms
of bibliometric
performance..."

A search in SCOPUS with subject headings relating to ecology and conservation between 2005 and 2015 was performed. In total 8.322 papers were included. The data extracted were citation counts and Altmetric score.
The results demonstrate that Altmetric score was positively correlated with citation counts.
Authors found strong association between science communication (measured by the Altmetric score) and citation counts. 


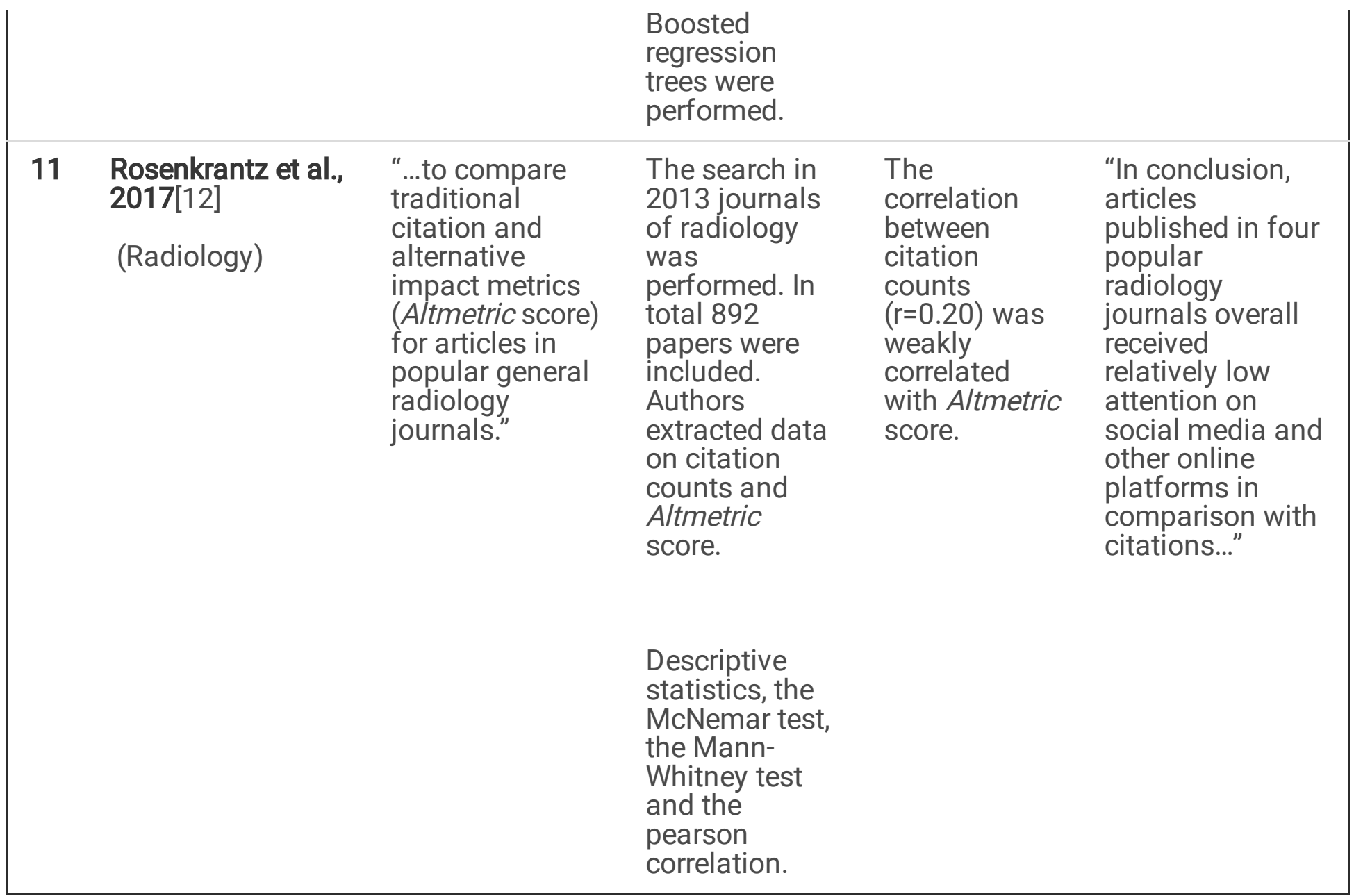

TABLE 2. Summary of the association between variables of the publishing journal and the publishing articles with Altmetric scores 


\begin{tabular}{|c|c|c|c|c|c|c|}
\hline $\begin{array}{l}\text { Studies and } \\
\text { analyses }\end{array}$ & $\begin{array}{l}\text { Citation } \\
\text { counts }\end{array}$ & $\begin{array}{l}\text { Journal } \\
\text { impact } \\
\text { factor }\end{array}$ & $\begin{array}{l}\text { Access } \\
\text { counts }\end{array}$ & $\begin{array}{l}\text { Open } \\
\text { access }\end{array}$ & $\begin{array}{l}\text { Time since } \\
\text { publication }\end{array}$ & $\begin{array}{l}\text { Press } \\
\text { release }\end{array}$ \\
\hline \multicolumn{7}{|c|}{ Correlation analysis } \\
\hline $\begin{array}{l}\text { Amath et al., } \\
\text { 2017[14] }\end{array}$ & $r=0.25$ & & $r=0.30$ & & & \\
\hline $\begin{array}{l}\text { Barbic et al., } \\
2016[21]\end{array}$ & $r=0.22$ & $r=0.35$ & & & & \\
\hline $\begin{array}{l}\text { Costas et al., } \\
2015[16]\end{array}$ & $r=0.18$ & $r=0.19$ & & & & \\
\hline $\begin{array}{l}\text { Delli et al., } \\
\text { 2017[22] }\end{array}$ & $r=0.27$ & & & & $r=-0.25$ & \\
\hline $\begin{array}{l}\text { Knight, } \\
2014[19]\end{array}$ & $\begin{array}{l}r=0.16^{*} \\
\text { and } \\
r=0.23^{\star *}\end{array}$ & & & & & \\
\hline $\begin{array}{l}\text { Rosenkrantz et } \\
\text { al., 2017[12] }\end{array}$ & $r=0.20$ & & & & & \\
\hline \multicolumn{7}{|c|}{ Multivariate regression analysis } \\
\hline $\begin{array}{l}\text { Araujo et al., } \\
2018[13]\end{array}$ & $\begin{array}{l}\beta=5.2^{\star} \text { and } \\
\beta=10.1^{\star \star}\end{array}$ & $\beta=3.4$ & & & $\beta=-4.9$ & \\
\hline $\begin{array}{l}\text { Haneef et al., } \\
2017[18]\end{array}$ & & $\mathrm{RoM}=1.10$ & & RoM=1.48 & & RoM=10.14 \\
\hline \multicolumn{7}{|c|}{ Boosted regression trees analysis } \\
\hline \multicolumn{7}{|c|}{ Lamb et al., 2018[20] Altmetric scores was strongly associated with citation counts. } \\
\hline \multicolumn{7}{|c|}{ Principal component analysis and factor analysis } \\
\hline \multicolumn{7}{|c|}{ Bornmann et al., 2018[15] Altmetric reader score are associated to citation counts. } \\
\hline
\end{tabular}

*These numbers represent $A$ Altmetric mentioned. ${ }^{*}$ These numbers represent $A$ Altmetric reader. $\mathrm{r}=$ Correlation estimates. RoM= Regression coefficients represent the logarithm of ratio of mean. $\beta=\beta$ coefficient.

\section{Figures}




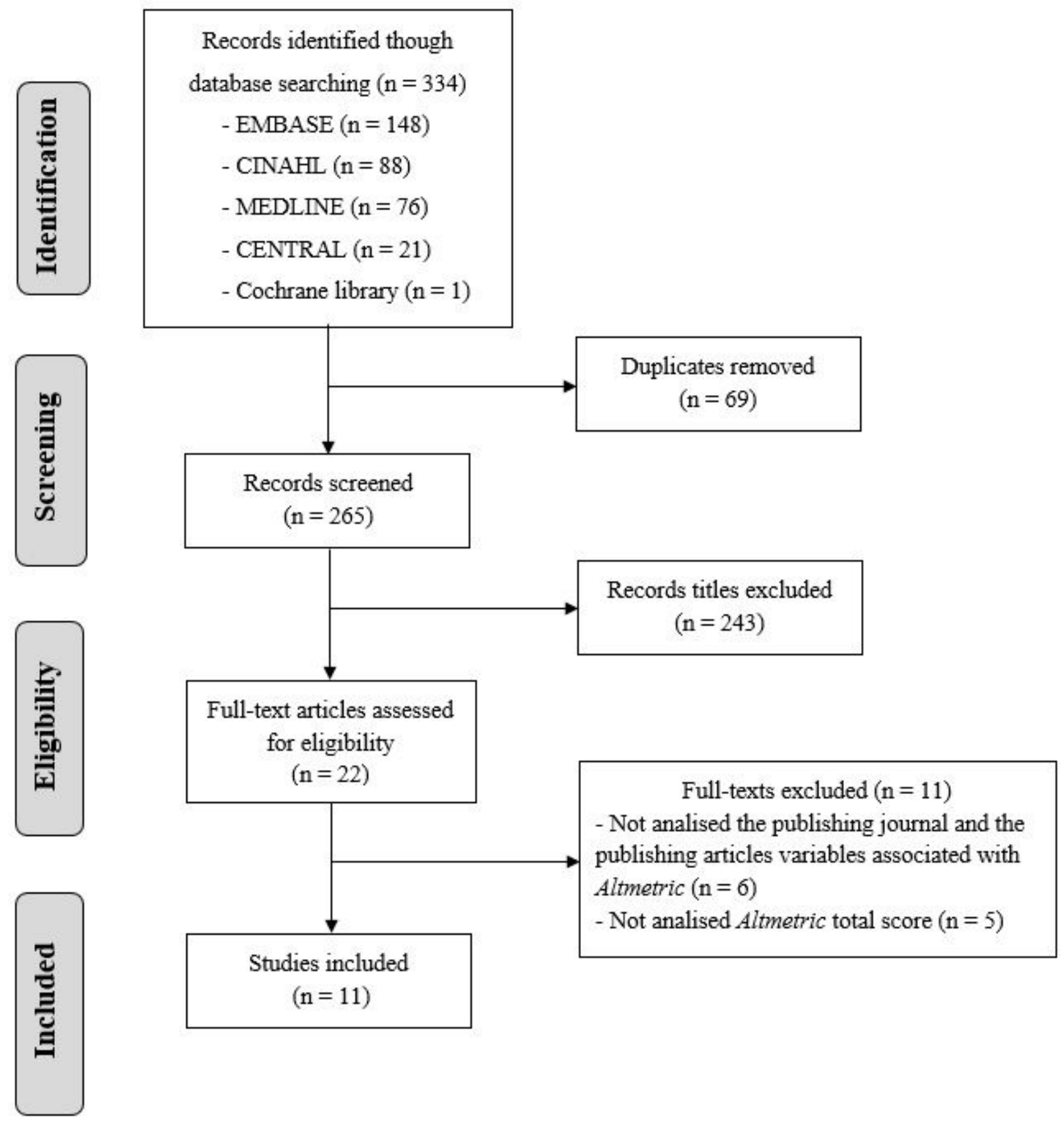

\section{Figure 1}

Study flow diagram of the eligibility assessment.

\section{Supplementary Files}

This is a list of supplementary files associated with this preprint. Click to download. 
- PRISMAPchecklist.docx 